International Journal of Pure and Applied Mathematics

Volume 99 No. 2 2015, 145-151

ISSN: 1311-8080 (printed version); ISSN: 1314-3395 (on-line version)

url: http://www.ijpam.eu

doi: http://dx.doi.org/10.12732/ijpam.v99i2.2

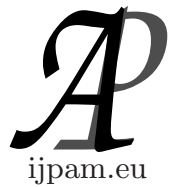

\title{
SYMMETRIC FORM OF THE VON NEUMANN POKER MODEL
}

\author{
Guido David ${ }^{1}$, Pearl Anne Po ${ }^{2}$ \\ ${ }^{1,2}$ Institute of Mathematics \\ National Sciences Research Institute \\ University of the Philippines \\ Quezon City, 1101, PHILIPPINES
}

\begin{abstract}
The von Neumann poker model, a two person zero-sum game, is a simplified poker simulation that can be used to analyze optimal betting and bluffing plays of the first player. The symmetric form is an extension of the von Neumann that allows both players to bet, making it a more realistic model of poker. The symmetric form is analyzed using Game Theory and best response, and the result is a characterization of the optimal betting and bluffing strategies and bet sizing of each player.
\end{abstract}

AMS Subject Classification: 91A05, 91A60, 91A24

Key Words: game theory, two player games, zero-sum game, von Neumann poker model, continuous poker model

\section{Introduction}

High Card Poker is a simple betting game that can be viewed as a model of poker situations [10,9]. In this game, each player is dealt one card after committing an ante in the pot. A round of betting ensues, and in the event of a showdown, which occurs when a bet is called, the cards are shown and the

Received: September 10, 2014

(C) 2015 Academic Publications, Ltd.

${ }^{\S}$ Correspondence author url: www.acadpubl.eu 
player with the higher card wins. Using a standard 52-card deck, the highest card is the Ace, the lowest the Deuce, with ties if the cards are of equal rank. In the more general case, the player is simply given a random number between 0 and $1[2,4,13]$.

We consider here a game with only two players. The initial ante is $\$ 1$ per player. In the von Neumann model [12], Player I acts first by betting an amount $\alpha$ or checking. If Player I bets, Player II has the option to call or fold. If Player I checks, Player II checks as well, resulting in a showdown. The von Neumann model is more realistic than the Borel model [3]. In the poker endgame, Player I is dealt a fixed value known to both players [5], in the Kuhn Poker Model [7], this is typically a Jack or a value of 0.75 . [10,9].

In extensions to the von Neumann model, reraises are allowed $[4,14]$, however, no action occurs after Player I checks. In this model, referred here as Symmetric von Neumann model, after Player I checks, Player II has the option to bet an amount $\beta$ or check. A bet by Player II gives Player I the option to call or fold, while a check results in a showdown. The extended form is a more realistic model of poker, because it allows both players the same betting options. The model is applicable to river (last card) situations in Texas Holdem, Seven Card Stud and Omaha [11], where hand rankings follow the Well Ordering Principle. Pre-flop play most poker games may be modeled using flip [1]. Note that the symmetric von Neumann model of Goldman and Stone [6] is different in that their model requires the players to act simultaneously, thereby eliminating positional advantage, and players may "drop" or fold rather than checking.

\section{The von Neumann Model}

Let the value dealt to Player I and II be $x$ and $y$ respectively, where $0 \leq x, y, \leq 1$. A viable strategy for Player I is $\{m\}$, i.e. betting whenever $x>m$ and checking otherwise. Let Player II's calling strategy be given by $\{n\}$, wherein Player II calls whenever $y>n$ and folds otherwise. The expected payoff $P$ from Player I's perspective given the strategy pair $\{m\},\{n\}$ is

$$
P= \begin{cases}\alpha(1-m)(m-n), & n \leq m, \\ (n+\alpha n-\alpha-m)(n-m), & n \geq m .\end{cases}
$$

Because Player I acts first, Player II optimizes her payoff using best response, by minimizing the expectation of Player I. This is given by

$$
n=\frac{(\alpha+2) m+\alpha}{2(\alpha+1)} \text {. }
$$


Substituting (2) in (1) over the interval $[m, 1]$ gives

$$
P=-\frac{\alpha^{2}(1-m)^{2}}{4(\alpha+1)} .
$$

Thus Player II should call tighter than the betting range of Player I. From (3), Player I's best strategy is $m=1$, or to check all hands (possibly betting $x=1$ ) for a value $V=0$. Player I does not seem to have the advantage. But if Player II is calling with a tighter range, it must be correct for Player I to bet with some of her worst hands, which do not have showdown value, as part of a balanced strategy. Denote this strategy by $\{m, r\}$ where $r<m$, i.e. Player I bets when $x>m$ or when $x \leq r$. This strategy is said to be polarized in poker lingo, wherein Player I bets with the best hands for value and the worst hands as a bluff. There is no benefit to calling with weak hands, thus Player II uses the calling strategy $\{n\}$. The payoff is given by

$$
P= \begin{cases}(1+\alpha) n^{2}+\alpha(1-m)(m-n)-\alpha r(1+n-r), & n \in[0, r], \\ n r(2+\alpha)-r(\alpha+r)+\alpha(1-m)(m-n), & n \in[r, m], \\ \alpha(m-n-r)(1-n)+(n-m)^{2}+r(2 n-r), & n \in[m, 1] .\end{cases}
$$

$P$ is continuous and (4) is linear in $n$ over $[r, m]$, hence the minimum payoff occurs at the interior points $r, m$, the endpoints $0, m$ or in the intervals $[0, r]$ or $[m, 1]$. From [13],

Theorem 1. In the von Neumann model, Player I's optimal strategy is to bet with $x>m$ or $x \leq r$, given by

$$
\begin{gathered}
m=\frac{\alpha^{2}+4 \alpha+2}{(\alpha+4)(\alpha+1)}, \\
r=\frac{\alpha}{(\alpha+4)(\alpha+1)} .
\end{gathered}
$$

Player II's best response is $n \in[r, m]$. The value of the game is positive and equal to

$$
V=\frac{\alpha}{(\alpha+4)(\alpha+1)} .
$$

Example 1. In High Card Poker, when the bet amount $\alpha=1$, Player I bets with $\{A, K, Q, 2\}$ for a value of 0.095 .

Corollary 2. The optimal bet size for Player $I$ is $\alpha=2$. Player I then bets when $x>7 / 9$ or $x \leq 1 / 9$ and checks the rest of the time. Player $I$ is indifferent to Player II's strategy, who calls with $y \in[r, m]$. The value of the game is $V=1 / 9$. 


\section{Symmetric von Neumann Model}

Consider now an extension to the von Neumann model. Player I has the option to check or bet $\alpha$ as before. If Player I bets, Player II may call or fold. If Player I checks, Player II may opt to check which leads to showdown, or bet an amount $\beta$, to which Player I either calls or folds. The basic von Neumann model implies the feasible strategies to be used in the symmetric form.

As before, let Player I's betting strategy be given by $\{m, r\}$. Player II's calling strategy does not depend on her betting strategy, so let it be given by $\{n\}$. After Player I checks with $x \in(r, m]$, let Player II's betting strategy be given by $\{u, t\}$, i.e. $y>u$ or $y \leq t$. Player I's calling strategy in response to a bet is to call when $x>s$.

Lemma 3. Given Player I's strategy $\{m, r, s\}$ and Player II's strategy $\{n, u, t\}$ in the Symmetric von Neumann game, then

(i) $r \leq n \leq m, t \leq s \leq u$;

(ii) $u \leq m, t \geq r$.

Although the above can be proved mathematically, we give a logical argument instead. From Theorem 1, the calling strategies (i) both follow. If $u>m$, then Player II is not betting some hands that beat Player I's checking range, while if $t<r$, then Player II is checking back some hands which cannot win at showdown, hence (ii). The payoff of the extended von Neumann model given strategies $\{m, r, s\}$ and $\{n, u, t\}$ satisfying Lemma 3 is

$$
\begin{aligned}
P=a((1-m)(m-n)-(1-n) r)+ & b\left(m^{2}-(m-s)(1-t)\right) \\
& -b u(m+s-u)-2(s t-n r)+t^{2} .
\end{aligned}
$$

Theorem 4. In an symmetric von Neumann game where Player I uses a betting strategy $\{m, r\}$ with bet size $\alpha$ and calling strategy $\{s\}$, Player II uses a calling strategy $\{n\}$ and a betting strategy $\{u, t\}$ with bet size $\beta$, then the strategies are optimal when they satisfy Lemma 3 with $r$ given by (8) and $u, t$ given by

$$
\begin{aligned}
& u=\frac{m(\beta+2)(\beta+1)+\beta}{(\beta+4)(\beta+1)}, \\
& t=\frac{\beta(\beta+2)-m \beta(\beta+1)}{(\beta+4)(\beta+1)} .
\end{aligned}
$$


Proof. To obtain Player I's best response to Player II's bet, we solve $\frac{d P}{d s}=0$ to get

$$
t=\frac{\beta(1-u)}{\beta+2} .
$$

To get Player II's optimal betting strategy, substitute (11) into (8) and solve $\frac{d P}{d u}=0$ for $u$ to obtain (9), while (11) leads to (10).

Notice that Player II's bluffing range (11) has the same form as Player I's bluffing range (6). The result is Player I is indifferent to Player II's calling strategy $\{n\}$ and Player II is indifferent to Player I's calling strategy $\{s\}$.

Corollary 5. In the extended von Neumann game, Player I's optimal betting strategy is given by $\{m, r\}$ where $r$ is given by (6) and

$$
m=\frac{4 \alpha(\alpha+1)+\beta\left(\alpha^{2}-\alpha-4\right)}{4 \alpha(\alpha+2)+\beta(\alpha+2)(\alpha-3)} .
$$

Proof. We obtain (6) by solving $\frac{d P}{d n}=0$ for $r$. Substituting (12), (9) and (10) in $P$ and solving $\frac{d P}{d m}=0$ for $m$ leads to the result.

Given that the bet amounts $\alpha, \beta$ are fixed, then the optimal strategies for both players are completely determined.

Example 2. If $\alpha=\beta=1$, then $m=2 / 3, r=1 / 9, u=1 / 2, t=1 / 6$, with $n \in[r, m]$ and $s \in[t, u]$ and $V=-1 / 18$.

If the players can choose their bet sizes, for example in no-limit poker games, then we must first determine Player II's optimal bet sizing. One strategy for Player II is to choose $\beta=2 \alpha$ which leads to $m=1$ in (12). The implication of this is that Player I checks back every hand, possibly with the exception $x=1$ (to which Player II can respond correctly). This essentially transposes the game back to the basic von Neumann model, with Player II now as the player with the option to check or bet. The expected payoff using Corollary 2 is $P=-1 / 9$. However, Player I can do better. Using the optimal strategies and solving $\frac{d P}{d b}=0$,

$$
\beta=\frac{2 \alpha(\alpha+5)}{\alpha^{2}-\alpha+6}
$$

Substituting this in (8) and solving $\frac{d P}{d \alpha}=0$ leads to the following result: 
Theorem 6. In the extended von Neumann game, if the two players are allowed to choose their bet sizes, the optimal bet for Player I is $\alpha=(5+\sqrt{57}) / 2$. The best response for Player II is $\beta=(33+5 \sqrt{57}) /(12+\sqrt{57})$. The value of the extended von Neumann game is $V=-0.0862859$.

Note that Player I's optimal bet amount is $\alpha=6.275$, more than three times the pot. This causes Player II to bet smaller rather than larger, with $\beta=3.619$. Player I's betting strategy is $x>0.942, x \leq 0.044$, and Player II's betting strategy is $y>0.797, y \leq 0.131$.

\section{Conclusion}

The extension to the von Neumann model allows Player II to bet some amount $\beta$ after Player I checks. Just as with the von Neumann model, this game was solved completely for optimal strategies. If the bet amounts were prescribed or fixed, both players used a polarized betting strategy of betting with the best hands and some of the worst hands based on Theorem 4 and Corollary 5 . If the players may choose their bet sizes, the optimal bet size for Player I is $\alpha=6.27$, betting the top $5.8 \%$ of hands and the bottom $4.4 \%$. If Player I checks, the optimal bet size of Player II is $\beta=3.62$, betting with the top $80 \%$ of hands and the bottom 13\%. Although the symmetric form of the von Neumann model more accurately describes poker, reraises $[4,14]$ were not considered, though these may be incorporated in the model. Another possibility is to model the game for three players [8]. Against most typical players who reraise predictably, the results of this paper may be used profitably in poker games.

\section{Acknowledgments}

This project was supported by the National Sciences Research Institute of the University of the Philippines, Project Reference No. 2008.149.

\section{References}

[1] N. Bernasconi, J. Lorenz, R. Spohel, Von Neumann and Newman poker with a flip of hand values, Discrete Math, 311 (2011), 2337-2345. doi:10.1016/j.disc.2011.05.033

[2] B. Chen, J. Ankenman, The Mathematics of Poker, Conjelco, USA (2006). 
[3] C. Ferguson, T.F. Ferguson, On the Borel and von Neumann poker models, In:Game Theory and Applications 9, Nova Sci. Publ., USA (2003).

[4] C. Ferguson, T.F. Ferguson, C. Gawargy, Uniform $(0,1)$ two-person poker models, In:Game Theory and Applications 12, Nova Sci. Publ., USA (2007).

[5] T.F. Ferguson, Two-person zero sum games, In:Game Theory, Second Edition, UCLA, USA (2014).

[6] A.J. Goldman, J.J. Stone, A symmetric continuous poker model, J. Res. Natl Bur. Stand., 64b:1 (1960), 35-40. doi:10.6028/jres.064B.004

[7] H.W. Kuhn, A simplified two-person poker, In:Contributions to the Theory of Games I, Princeton University Press, USA (1950), 97-104.

[8] J.F. Nash, L.S. Shapely, A simple three-person poker game, In:Contributions to the Theory of Games I, Princeton University Press, USA (1950), 105-116.

[9] J.D. Pascual, Optimal strategies for an extension of the von Neumann poker model, Thesis, University of the Philippines, Philippines (2013).

[10] A.P. Sambas, Evaluating the payoff matrix of the poker endgame, Thesis, University of the Philippines, Philippines (2010).

[11] D. Sklansky, The Theory of Poker, Two Plus Two Publishing, USA (1989).

[12] J. von Neumann, O. Morgenstern, Theory of Games and Economic Behavior, Princeton University Press, USA (1953), 186-219.

[13] C. Warmbrand, Best response to tight and loose opponents in the Borel and von Neumann poker models, Working Paper, University of Arizona, USA (2003). doi:10.1.1.178.1900

[14] H. Zhang, Exposition of two-player zero-sum poker games, Master's Thesis, University of Pennsylvania, USA (2010). 
